\title{
Fixed point theorems for contracting mappings in partial metric spaces
}

Lech Pasicki* $^{*}$

\section{"Correspondence:}

pasicki@agh.edu.pl

Faculty of Applied Mathematics,

AGH University of Science and

Technology, Al. Mickiewicza 30,

Kraków, 30-059, Poland

\begin{abstract}
The paper deals with a class of contracting mappings that includes the Boyd-Wong and the Matkowski classes. Some of the new fixed point theorems obtained here unify and extend well-known results also in the case of metric spaces.
\end{abstract}

MSC: 47H09; 47H10; 54H25; 54E99

Keywords: partial metric space; fixed point; multivalued mapping; generalized contraction

\section{Introduction}

In the simplest case, the condition with a contracting mapping has the form $d(f(y), f(x)) \leq$ $\varphi(d(y, x))$, where $(X, d)$ is a metric space, $f$ is a mapping on $X$, and $\varphi:[0, \infty) \rightarrow[0, \infty)$ is such that $\varphi(\alpha)<\alpha, \alpha>0$. Boyd and Wong [1] assumed that $\varphi$ is upper semicontinuous from the right (i.e., $\lim \sup _{\beta \rightarrow \alpha^{+}} \varphi(\beta) \leq \varphi(\alpha), \alpha \geq 0$ ); Matkowski in [2] assumed that $\varphi$ is nondecreasing and such that $\lim _{n \rightarrow \infty} \varphi^{n}(\alpha)=0$ for each $\alpha>0$. We assume that all sequences $\left(a_{n}\right)_{n \in \mathbb{N}}$ such that $a_{n+1} \leq \varphi\left(a_{n}\right), n \in \mathbb{N}$, converge to zero. It appears that the classes of Boyd-Wong's and Matkowski's mappings are included in this new class (the problem of $\varphi(0)$ for Boyd-Wong mappings is meaningless for contractions). The main results are Theorems 21, 22, 23, and theorems extending the well-known classical results: Theorem 30 (Matkowski's theorem) and Theorem 31 (covering the theorems of Romaguera and BoydWong).

Let us recall the notions of a partial metric space due to Matthews [3, Definition 3.1] and of a dualistic partial metric due to Oltra and Valero [4] and O'Neill [5].

Definition 1 A dualistic partial metric is a mapping $p: X \times X \rightarrow \mathbb{R}$ such that

$$
\begin{aligned}
& y=x \quad \text { iff } \quad p(y, y)=p(y, x)=p(x, x), \quad x, y \in X \\
& p(y, y) \leq p(y, x), \quad x, y \in X \\
& p(y, x)=p(x, y), \quad x, y \in X \\
& p(z, x) \leq p(z, y)+p(y, x)-p(y, y), \quad x, y, z \in X .
\end{aligned}
$$

If $p$ is nonnegative, then it is a partial metric.

(02014 Pasicki; licensee Springer. This is an Open Access article distributed under the terms of the Creative Commons Attribution License (http://creativecommons.org/licenses/by/2.0), which permits unrestricted use, distribution, and reproduction in any medium, provided the original work is properly cited. 
If $p$ is a dualistic partial metric on $X$, then $q: X \times X \rightarrow[0, \infty)$ defined by

$$
q(y, x)=p(y, x)-p(y, y), \quad x, y \in X
$$

is a quasi-metric [3, Theorem 4.1] ( $y=x$ iff $q(y, x)=q(x, y)=0, q(z, x) \leq q(z, y)+q(y, x))$.

An open ball for $x \in X, \epsilon>0$ is defined by

$$
B(x, \epsilon)=\{y \in X: q(x, y)<\epsilon\}=\{y \in X: p(x, y)<p(x, x)+\epsilon\} .
$$

The family of open balls generates topology $\mathcal{T}_{q}$ on $X$. It is accepted that the (dualistic) partial metric space $(X, p)$ is equipped with the topology $\mathcal{T}_{q}$.

It is known (see, e.g., [4]) that a metric $d$ can be defined by a dualistic partial metric $p$ as follows:

$$
\begin{aligned}
d(y, x) & =\max \{q(y, x), q(x, y)\} \\
& =\max \{p(y, x)-p(y, y), p(x, y)-p(x, x)\}, \quad x, y \in X .
\end{aligned}
$$

In this paper it is understood that $q, d$ are defined by (5), (7) respectively for a (dualistic) partial metric $p$.

For dualistic partial metric spaces, it is accepted (see, e.g., [4, p.19]) that $\left(x_{n}\right)_{n \in \mathbb{N}}$ is called a Cauchy sequence in $(X, p)$ if $\lim _{m, n \rightarrow \infty} p\left(x_{n}, x_{m}\right)=\alpha \in \mathbb{R}$, and $(X, p)$ is complete if for every Cauchy sequence $\left(x_{n}\right)_{n \in \mathbb{N}}$ in $(X, p)$, there exists $x \in \lim _{n \rightarrow \infty} x_{n}$ such that $\lim _{m, n \rightarrow \infty} p\left(x_{n}, x_{m}\right)=p(x, x)$.

The following notions are useful.

Definition 2 [6, Definition 2.1] Let $p: X \times X \rightarrow \mathbb{R}$ be a mapping. The kernel of $p$ is the set $\operatorname{Ker} p=\{x \in X: p(x, x)=0\}$.

Definition 3 (cp. [7, Definition 2.1]) A dualistic partial metric space $(X, p)$ is 0-complete if for every sequence $\left(x_{n}\right)_{n \in \mathbb{N}}$ in $X$ such that $\lim _{m, n \rightarrow \infty} p\left(x_{n}, x_{m}\right)=0$, there exists $x \in$ $\lim _{n \rightarrow \infty} x_{n} \cap \operatorname{Ker} p$ in $(X, p)$.

In fact Definition 3 is too abstract. The condition $x \in \lim _{n \rightarrow \infty} x_{n} \cap \operatorname{Ker} p$ in $(X, p)$ means that $\lim _{n \rightarrow \infty} p\left(x, x_{n}\right)=p(x, x)=0$ (see (6), (2)). If, in addition, $\lim _{m, n \rightarrow \infty} p\left(x_{n}, x_{m}\right)=0$, then $x=\lim _{n \rightarrow \infty} x_{n}$ in $(X, d)$ (see [4, Lemma 2.2] or [6, Proposition 1.4]).

Corollary 4 A dualistic partial metric space $(X, p)$ is 0 -complete iffevery sequence $\left(x_{n}\right)_{n \in \mathbb{N}}$ such that $\lim _{m, n \rightarrow \infty} p\left(x_{n}, x_{m}\right)=0$ converges in $(X, d)$ to a point $x \in \operatorname{Ker} p$. If $p$ is a metric, then 0 -completeness is identical with completeness.

There exist 0 -complete partial metric spaces which are not complete [8]. Some criterions of 0 -completeness can be found in [9, Section 4].

Proposition 5 Let $(X, p)$ be a partial metric space. Then $\left(x_{n}\right)_{n \in \mathbb{N}}$ converges in $(X, d)$ to $x \in \operatorname{Ker} p$ iff $\lim _{n \rightarrow \infty} p\left(x, x_{n}\right)=0$ and iff we have $x \in \lim _{n \rightarrow \infty} x_{n} \cap \operatorname{Ker} p$ in $(X, p)$. 
Proof A sequence $\left(x_{n}\right)_{n \in \mathbb{N}}$ converges to $x$ in $(X, d)$ iff $\lim _{m, n \rightarrow \infty} p\left(x_{n}, x_{m}\right)=\lim _{n \rightarrow \infty} p(x$, $\left.x_{n}\right)=p(x, x)$ (see [4, Lemma 2.2] or [6, Proposition 1.4]). Assume $\lim _{n \rightarrow \infty} p\left(x, x_{n}\right)=0$. Then, for nonnegative $p$, condition (2) yields $p(x, x)=0$ and $\lim _{n \rightarrow \infty} p\left(x, x_{n}\right)=p(x, x)$, i.e., $x \in \operatorname{Ker} p$ and $x \in \lim _{n \rightarrow \infty} x_{n}$ in $(X, p)$. We also have

$$
0 \leq p\left(x_{n}, x_{m}\right) \leq p\left(x_{n}, x\right)+p\left(x, x_{m}\right)-p(x, x)=p\left(x, x_{n}\right)+p\left(x, x_{m}\right)
$$

and consequently, $\lim _{m, n \rightarrow \infty} p\left(x_{n}, x_{m}\right)=\lim _{n \rightarrow \infty} p\left(x, x_{n}\right)=p(x, x)=0$, i.e., $x \in \operatorname{Ker} p$ and $\left(x_{n}\right)_{n \in \mathbb{N}}$ converges in $(X, d)$ to $x$.

Let $2^{Y}$ be the family of all subsets of $Y$. We say that $F: X \rightarrow 2^{Y}$ is a (multivalued) mapping if $F(x) \neq \emptyset$ for all $x \in X \neq \emptyset$.

Now, let us investigate the concept of a 0 -closed graph.

Definition 6 Let $(X, p)$ be a dualistic partial metric space. A mapping $F: X \rightarrow 2^{X}$ has a 0 -closed graph if for all sequences $\left(x_{n}\right)_{n \in \mathbb{N}},\left(y_{n}\right)_{n \in \mathbb{N}}$ in $X$ the following condition is satisfied:

$$
\lim _{n \rightarrow \infty} p\left(x, x_{n}\right)=\lim _{n \rightarrow \infty} p\left(x, y_{n}\right)=0, \quad y_{n} \in F\left(x_{n}\right), n \in \mathbb{N}, \text { and } x \in \operatorname{Ker} p \text { yield } x \in F(x) .
$$

Proposition 5 and [6, Proposition 1.4] yield the following.

Corollary 7 For all sequences $\left(x_{n}\right)_{n \in \mathbb{N}},\left(y_{n}\right)_{n \in \mathbb{N}}$ with $\lim _{m, n \rightarrow \infty} p\left(x_{n}, x_{m}\right)=\lim _{m, n \rightarrow \infty} p\left(y_{n}\right.$, $\left.y_{m}\right)=0$, condition (8) can be replaced by

$$
\lim _{n \rightarrow \infty} x_{n}=\lim _{n \rightarrow \infty} y_{n}=x \quad \text { in }(X, d), y_{n} \in F\left(x_{n}\right), n \in \mathbb{N}, \text { and } x \in \operatorname{Ker} p \text { yield } x \in F(x) .
$$

If $p$ is nonnegative, then (8) is equivalent to (9).

Clearly, if $F: X \rightarrow 2^{X}$ has a closed graph in $(X, d) \times(X, d)$, then (9) is satisfied as $\operatorname{Ker} p$ with topology induced by $p$ is a closed metric subspace of $(X, d)$ [6, Lemma 2.2].

For a partial metric space $(X, p)$, a nonempty set $A \subset X$, and $z \in X$, let us adopt

$$
p(A, z)=\inf \{p(x, z): x \in A\}
$$

and

$$
E(A, r)=\{z \in X: p(A, z)<r\} .
$$

The family $\left\{E(A, \epsilon): A \in 2^{X} \backslash\{\emptyset\}, \epsilon>0\right\}$ generates a topology $\mathcal{P}$, and we get a topological space $\left(2^{X} \backslash\{\emptyset\}, \mathcal{P}\right)$. Assume that $p$ is nonnegative and

for every $\epsilon>0$, there exists $\delta>0$ such that for each

$$
u \in X, p(x, u)<\delta \text { yields } F(u) \subset E(F(x), \epsilon)
$$

holds. Then, for all sequences $\left(x_{n}\right)_{n \in \mathbb{N}},\left(y_{n}\right)_{n \in \mathbb{N}}$ such that $\lim _{n \rightarrow \infty} p\left(x, x_{n}\right)=\lim _{n \rightarrow \infty} p(x$, $\left.y_{n}\right)=0$ and $y_{n} \in F\left(x_{n}\right), n \in \mathbb{N}$, there exist $z_{n} \in F(x), n \in \mathbb{N}$ with $\lim _{n \rightarrow \infty} p\left(y_{n}, z_{n}\right)=0$. We have

$$
p\left(x, z_{n}\right) \leq p\left(x, y_{n}\right)+p\left(y_{n}, z_{n}\right)
$$


and consequently, $x=\lim _{n \rightarrow \infty} z_{n}$ in $(X, d)$ (Proposition 5). If $F(x) \cap \operatorname{Ker} p=\overline{F(x)} \cap \operatorname{Ker} p$ in $(X, d)$, then $x \in F(x)$ holds.

For a partial metric space $(X, p)$ and nonempty $A, C \subset X$, let us adopt

$$
P(A, C)=\inf \{r>0: A \subset E(C, r) \text { and } C \subset E(A, r)\} .
$$

If $p$ is a metric, then $P$ is the Hausdorff metric of the metric $p$, whenever $A$ and $C$ are nonempty closed and bounded subsets of $X$. In general, $P$ is not a partial metric (see [10, Proposition 2.2(i), Proposition 2.3(h3)]).

Clearly, $p(x, u)-p(x, x)=p(x, u)$ for $x \in \operatorname{Ker} p$ and if (10) holds, then $F:(X, p) \rightarrow\left(2^{X} \backslash\right.$ $\{\emptyset\}, \mathcal{P})$ is continuous on $\operatorname{Ker} p$.

Corollary 8 Let $(X, p)$ be a partial metric space. Assume that for each $x \in \operatorname{Ker} p$, the mapping $F: X \rightarrow 2^{X}$ satisfies (10) and $F(x) \cap \operatorname{Ker} p=\overline{F(x)} \cap \operatorname{Ker} p$ in $(X, d)$. Then $F$ has a 0 -closed graph (and conditions (8), (9) are equivalent).

Proposition 9 Let $(X, p)$ be a dualistic partial metric space. If $F: X \rightarrow 2^{X}$ has a 0 -closed graph, then Fix $F \cap \operatorname{Ker} p$ is closed in $(X, d)$.

Proof If $x_{n} \in \operatorname{Fix} F \cap \operatorname{Ker} p, n \in \mathbb{N}$, then for $y_{n}=x_{n}, n \in \mathbb{N}$ and each $x \in \operatorname{Ker} p$ such that $\lim _{n \rightarrow \infty} p\left(x, x_{n}\right)=0$, condition (8) yields $x \in F(x)$, i.e., Fix $F \cap \operatorname{Ker} p$ is closed in $\operatorname{Ker} p$ (which with topology induced by $p$ is a closed metric subspace of $(X, d)$ [6, Lemma 2.2]).

Now, let us investigate a 'contraction' condition.

Let $\boldsymbol{\Phi}$ be a class of mappings $\varphi:[0, \infty) \rightarrow[0, \infty)$ such that $\varphi(\alpha)<\alpha, \alpha>0 ; \varphi \in \boldsymbol{\Phi}_{0}$ iff $\varphi \in \Phi$ and $\varphi(0)=0$.

Proposition 10 Let $(X, p)$ be a partial metric space. Assume that $G, H: X \rightarrow 2^{X}$ satisfy

$$
p(H(y), y) \leq \varphi(p(y, x)), \quad y \in G(x), x \in X
$$

for $\varphi \in \Phi_{0}$. Then Fix $G \subset \operatorname{Ker} p$; if $H$ has a 0 -closed graph, then Fix $G \subset$ Fix $H$ holds.

Proof For $y=x \in G(x)$ and nonnegative $p$, conditions (2), (12) yield

$$
0 \leq p(x, x) \leq \inf \{p(z, x): z \in H(x)\}=p(H(x), x) \leq \varphi(p(x, x))
$$

i.e., $p(x, x)=0$ and $x \in \operatorname{Ker} p$. What is more, from our inequality it follows that there exist $y_{n} \in H(x)$ such that $\lim _{n \rightarrow \infty} p\left(y_{n}, x\right)=0$. Now, (8) for $x_{n}=x, F=H$ yields $x \in H(x)$.

Propositions 9, 10 yield the following.

Theorem 11 Let $(X, p)$ be a 0 -complete partial metric space, and let $\mathcal{F}$ be a family of mappings $X \rightarrow 2^{X}$ with 0 -closed graphs. Assume that $\varphi \in \Phi_{0}$; some and at least all different $G, H \in \mathcal{F}$ satisfy (12). Then all members of $\mathcal{F}$ have the same set of fixed points; this set is closed in $(X, d)$ and contained in $\operatorname{Ker} p$. 
The previous result becomes a little more interesting if a mapping $F \in \mathcal{F}$ has a fixed point.

Let us assume that the following condition is satisfied for a partial metric space $(X, p), P$ defined by (11), and $G, H: X \rightarrow 2^{X}$ :

$$
P(H(y), G(x)) \leq \varphi(p(y, x)), \quad x, y \in X .
$$

Then, for any $y \in G(x)$, we have

$$
\begin{aligned}
p(H(y), y) & \leq \sup \{p(H(y), u): u \in G(x)\} \\
& \leq \max \{\sup \{p(H(y), u): u \in G(x)\}, \sup \{p(G(x), v): v \in H(y)\}\} \\
& =P(H(y), G(x)) \leq \varphi(p(y, x)),
\end{aligned}
$$

i.e., condition (13) yields condition (12). The subsequent two propositions enable us to strengthen condition (12).

Proposition 12 Let $(X, p)$ be a partial metric space, and let $C \subset X$ be compact in $(X, d)$. Then, for any $y \in X$, there exists $x \in C$ such that $p(x, y)=p(C, y)$.

Proof Let $\left(x_{n}\right)_{n \in \mathbb{N}}$ be a sequence in $C$ such that

$$
\lim _{n \rightarrow \infty} p\left(x_{n}, y\right)=\inf \{p(z, y): z \in C\}=p(C, y)=\alpha .
$$

There exists a subsequence $\left(x_{k_{n}}\right)_{n \in \mathbb{N}}$ of $\left(x_{n}\right)_{n \in \mathbb{N}}$ and $x \in C$ such that $x=\lim _{n \rightarrow \infty} x_{k_{n}}$ in $(X, d)$, i.e., [4, Lemma 2.2]

$$
\lim _{m, n \rightarrow \infty} p\left(x_{k_{n}}, x_{k_{m}}\right)=\lim _{n \rightarrow \infty} p\left(x, x_{k_{n}}\right)=p(x, x) .
$$

Now, from

$$
\alpha \leq p(x, y) \leq p\left(x, x_{k_{n}}\right)+p\left(x_{k_{n}}, y\right)-p\left(x_{k_{n}}, x_{k_{n}}\right)
$$

we get

$$
\alpha \leq p(x, y) \leq p(x, x)+\alpha-p(x, x)=\alpha .
$$

Corollary 13 Let $(X, p)$ be a partial metric space, and let $G, H: X \rightarrow 2^{X}$ be mappings with $H$ compact valued in $(X, d)$. Then condition (12) is equivalent to

$$
\begin{gathered}
\text { for each } x \in X, y \in G(x), \text { there exists } z \in H(y) \\
\text { such that } p(z, y)=p(H(y), y) \leq \varphi(p(y, x)) .
\end{gathered}
$$

Condition (14) extends the idea of $\alpha$-step mappings [11, Definition 17].

The next example shows that even a 'good' mapping $\varphi$ in condition (14) does not guarantee the existence of a fixed point. 
Example 14 Let us consider a continuous mapping $\varphi \in \Phi_{0}$ defined by $\varphi(\alpha)=\alpha /\left(\alpha^{2}+1\right)$ for $\alpha \in[0,1]$, and $\varphi(\alpha)=1 / 2$ for $\alpha>1$. An easy computation proves that $\varphi$ is increasing on $[0,1]$ and, therefore, $\varphi$ is nondecreasing on $[0, \infty)$. Let us consider $X=[1, \infty)$ and $f(x)=x+1 / x$, $x \in X$. For $p(y, x)=d(y, x)=|y-x|$, we have

$$
d\left(f^{2}(x), f(x)\right)=x+1 / x+1 /(x+1 / x)-x-1 / x=x /\left(1+x^{2}\right)=(1 / x) /\left((1 / x)^{2}+1\right) .
$$

Now, for $\alpha=d(f(x), x)=1 / x \leq 1$, we obtain

$$
d\left(f^{2}(x), f(x)\right)=\varphi(\alpha)=\varphi(d(f(x), x)),
$$

i.e., (14) is satisfied for $H=f, y=f(x), z=f^{2}(x)$. Still it is clear that $f: X \rightarrow X$ has no fixed point.

It is a good idea suggested by [12] to gather together the properties of $\varphi$. Let us recall that $\Phi$ is a class of mappings $\varphi:[0, \infty) \rightarrow[0, \infty)$ such that $\varphi(\alpha)<\alpha, \alpha>0$; and $\varphi \in \Phi_{0}$ iff $\varphi \in \Phi$ and $\varphi(0)=0$.

Proposition 15 Assume that $\varphi \in \Phi_{0}$. Then every sequence $\left(a_{n}\right)_{n \in \mathbb{N}}$ such that $a_{n+1} \leq \varphi\left(a_{n}\right)$, $n \in \mathbb{N}$ (in particular $\left.\left(\varphi^{n}(\alpha)\right)_{n \in \mathbb{N}}, \alpha \geq 0\right)$ is nonincreasing; if, in addition, $\varphi$ is nondecreasing, then $a_{n+1} \leq \varphi^{n}\left(a_{1}\right), n \in \mathbb{N}$ holds.

Proof From $\varphi(\alpha) \leq \alpha, \alpha \geq 0$, it follows that $a_{n+1} \leq \varphi\left(a_{n}\right) \leq a_{n}$. Similarly, for nondecreasing $\varphi$, we get $a_{2} \leq \varphi\left(a_{1}\right), a_{3} \leq \varphi\left(a_{2}\right) \leq \varphi^{2}\left(a_{1}\right), \ldots, a_{n+1} \leq \varphi^{n}\left(a_{1}\right)$.

Let us present some subclasses of $\Phi_{0}$.

Let $\boldsymbol{\Phi}_{P}$ consist of mappings $\varphi:[0, \infty) \rightarrow[0, \infty)$ for which every sequence $\left(a_{n}\right)_{n \in \mathbb{N}}$ such that $a_{n+1} \leq \varphi\left(a_{n}\right), n \in \mathbb{N}$ converges to zero.

Proposition 16 We have $\Phi_{P} \subset \Phi_{0}$. For $\varphi \in \Phi_{P}$, the sequence $\left(\varphi^{n}(\alpha)\right)_{n \in \mathbb{N}}$ is nonincreasing and it converges to $0, \alpha \geq 0$. If a mapping $\varphi \in \Phi_{0}$ satisfies

$$
\limsup _{\beta \rightarrow \alpha^{+}} \varphi(\beta)<\alpha, \quad \alpha>0,
$$

then $\varphi \in \Phi_{P}$.

Proof Assume that $\varphi \in \Phi_{P}$. Suppose that $\alpha \leq \varphi(\alpha)$ for $\alpha>0$. Then $a_{n}=\alpha, n \in \mathbb{N}$, is a good counterexample (the sequence does not converge to 0 ). Therefore $\Phi_{P} \subset \Phi$ holds. Suppose $\varphi(0)=a>0$. Then, for $a_{2 n-1}=a, a_{2 n}=0, n \in \mathbb{N}$, we obtain a divergent sequence $\left(a_{n}\right)_{n \in \mathbb{N}}$ such that $a_{n+1} \leq \varphi\left(a_{n}\right), n \in \mathbb{N}$. Consequently, every $\varphi \in \Phi_{P}$ satisfies $\varphi(0)=0$. The sequence $\left(\varphi^{n}(\alpha)\right)_{n \in \mathbb{N}}$ is nonincreasing, $\varphi^{n+1}(\alpha)=\varphi\left(\varphi^{n}(\alpha)\right) \leq \varphi^{n}(\alpha), n \in \mathbb{N}$ and it converges to 0 as $\varphi \in \Phi_{P}$. Assume $\varphi \in \Phi_{0}$. Then any sequence $\left(a_{n}\right)_{n \in \mathbb{N}}$ such that $a_{n+1} \leq \varphi\left(a_{n}\right)\left(\leq a_{n}\right)$, $n \in \mathbb{N}$ is nonincreasing and therefore it converges, say, to $\gamma \geq 0$. Suppose $\gamma>0$. Then (15) yields

$$
0=\lim _{n \rightarrow \infty}\left[a_{n+1}-a_{n}\right] \leq \limsup _{n \rightarrow \infty} \varphi\left(a_{n}\right)-\gamma<\gamma-\gamma=0
$$

a contradiction. 
Let $\boldsymbol{\Phi}_{B W} \subset \Phi_{0}$ consist of mappings $\varphi$ upper semicontinuous from the right, call them Boyd-Wong mappings. Proposition 16 yields $\Phi_{B W} \subset \Phi_{P}$.

In turn, let $\boldsymbol{\Phi}_{M}$ consist of nondecreasing mappings $\varphi:[0, \infty) \rightarrow[0, \infty)$ such that $\lim _{n \rightarrow \infty} \varphi^{n}(\alpha)=0, \alpha>0$ (Matkowski mappings). It is well known [13, Lemma] that $\Phi_{M} \subset \Phi_{0}$. Moreover, $a_{n+1} \leq \varphi\left(a_{n}\right), n \in \mathbb{N}$, yields $a_{n+1} \leq \varphi^{n}\left(a_{1}\right)$ (Proposition 15) and hence $\lim _{n \rightarrow \infty} a_{n}=0$. Consequently, $\Phi_{M} \subset \Phi_{P}$ holds. Let us note that $\varphi$ from Example 14 belongs to $\Phi_{M}$.

The following is a kind of the reverse condition.

Proposition 17 Let $\left(a_{n}\right)_{n \in \mathbb{N}}$ be a sequence convergent to zero and such that $a_{n+1} \leq \varphi\left(a_{n}\right)$, $n \in \mathbb{N}$, for $a \varphi \in \Phi_{0}$. Then there exists a mapping $\psi \in \Phi_{M} \cap \Phi_{B W}$ such that $\varphi\left(a_{n}\right) \leq \psi\left(a_{n}\right) \leq$ $a_{n}$, and $\psi^{n}(\alpha) \leq a_{n}, n \in \mathbb{N}, \alpha \geq 0$.

Proof Let us adopt $\psi(0)=0$ and $\psi(\alpha)=\sup \left\{\varphi\left(a_{n}\right): a_{n} \leq \alpha, n \in \mathbb{N}\right\}, \alpha>0$. Clearly, $\psi$ is nondecreasing and continuous from the right as $\lim _{n \rightarrow \infty} \varphi\left(a_{n}\right)=0$. Therefore, $\psi$ is also upper semicontinuous. The sequence $\left(a_{n}\right)_{n \in \mathbb{N}}$ is nonincreasing as $a_{n+1} \leq \varphi\left(a_{n}\right) \leq a_{n}, n \in \mathbb{N}$. Let us adopt $a_{0}=\infty$. For $0<a_{n} \leq \alpha<a_{n-1}$, we have

$$
\varphi\left(a_{n}\right) \leq \sup \left\{\varphi\left(a_{n-1+k}\right): k \in \mathbb{N}\right\}=\psi(\alpha)<\sup \left\{a_{n-1+k}: k \in \mathbb{N}\right\}=a_{n} \leq \alpha,
$$

and the case of $a_{n}=0$ is trivial as then $\psi(\alpha)=0<\alpha$. Consequently, we get $\psi(\alpha)<a_{1}$ (if $a_{1}>0$ ), $\psi^{2}(\alpha)<a_{2}$ (if $a_{2}>0$ ), and so on. Finally, we obtain $\psi^{n}(\alpha) \leq a_{n}, n \in \mathbb{N}$ (the possible case of some $a_{k}=0$ ).

The following modification of $\varphi$ is useful.

Proposition 18 Assume that $\varphi:[0, \infty) \rightarrow[0, \infty)$ is a mapping. Then $\psi$ defined by

$$
\psi(\alpha)=[\varphi(\alpha)+\alpha] / 2, \quad \alpha \geq 0,
$$

belongs to $\Phi$ iff $\varphi \in \Phi ; \varphi \in \Phi_{0}$ iff $\psi \in \Phi_{0} ; \varphi$ satisfies (15) iff $\psi$ satisfies (15). In addition, if $\varphi$ is nondecreasing, then $\psi$ is increasing.

Proof It is clear that $\varphi(\alpha)<\psi(\alpha)<\alpha$ holds iff $\psi(\alpha)<\alpha$. If $\varphi$ is nondecreasing, then for $0 \leq \alpha<\beta$ we obtain

$$
\psi(\beta)-\psi(\alpha)=[\varphi(\beta)-\varphi(\alpha)] / 2+(\beta-\alpha) / 2 \geq(\beta-\alpha) / 2>0,
$$

i.e., $\psi$ is increasing. The remaining part of the proof is also trivial.

The next two propositions can be helpful in proving fixed point theorems.

Proposition 19 Assume that $(X, p)$ is a partial metric space, and let $\varphi \in \Phi_{0}$. If mappings $G, H: X \rightarrow 2^{X}$ satisfy (12) and $H$ has a 0 -closed graph, then for $\varphi=\psi$, where $\psi$ is defined by (16), condition (14) holds. 
Proof For $p(y, x)>0$, there exist $\epsilon>0$ and $z \in H(y)$ such that $p(z, y) \leq \varphi(p(y, x))+\epsilon \leq$ $\psi(p(y, x))$. If $p(y, x)=0$, then (2) yields $x=y$, i.e., $x$ is a fixed point of $G$, and by Proposition $10, z=x \in H(x)$.

Proposition 20 Let $(X, p)$ be a partial metric space, let $\mathcal{F}$ be a nonempty family of at most two mappings $X \rightarrow 2^{X}$ with 0 -closed graphs, and let $\varphi \in \Phi_{P}$. Assume that all members of $\mathcal{F}$ either satisfy (12) and $\varphi$ has property (15) or satisfy (14). Then there exists a sequence $\left(x_{n}\right)_{n \in \mathbb{N}}$ such that $x_{2 n} \in H\left(x_{2 n-1}\right), x_{2 n+1} \in G\left(x_{2 n}\right), n \in \mathbb{N}$, and $\lim _{n \rightarrow \infty} p\left(x_{n+1}, x_{n}\right)=0$.

Proof If $\varphi \in \Phi_{0}$ satisfies (15) and (12) holds, then for $\psi$ as in (16) and $\varphi=\psi$, condition (14) is satisfied (Proposition 19), and $\varphi \in \Phi_{P}$ (Propositions 18, 16). Thus, it is sufficient to consider the case of $\varphi \in \Phi_{P}$ and condition (14). Let $x_{0} \in X$ be arbitrary, $x_{1} \in G\left(x_{0}\right)$, and let $x_{2} \in H\left(x_{1}\right)$ be such that $p\left(x_{2}, x_{1}\right) \leq \varphi\left(p\left(x_{1}, x_{0}\right)\right)$. If $x_{2 k} \in H\left(x_{2 k-1}\right)$ is defined, then $x_{2 k+1} \in G\left(x_{2 k}\right)$ is such that $p\left(x_{2 k+1}, x_{2 k}\right) \leq \varphi\left(p\left(x_{2 k}, x_{2 k-1}\right)\right)$, and, similarly, $x_{2 k+2} \in H\left(x_{2 k+1}\right)$ satisfies $p\left(x_{2 k+2}, x_{2 k+1}\right) \leq \varphi\left(p\left(x_{2 k+1}, x_{2 k}\right)\right)$. Thus, for $a_{n}=p\left(x_{n+1}, x_{n}\right)$, we have $a_{n+1} \leq \varphi\left(a_{n}\right)$, $n \in \mathbb{N}$, and $\left(a_{n}\right)_{n \in \mathbb{N}}$ converges to zero as $\varphi \in \Phi_{P}$.

As was shown in Example 14, conditions (12), (14) are too weak to guarantee the existence of a fixed point, even for $\varphi \in \Phi_{M}$. The next two theorems, with stronger assumptions, are general results.

Theorem 21 Let $(X, p)$ be a 0-complete partial metric space, and let $F: X \rightarrow 2^{X}$ be a mapping with 0 -closed graph. Assume that for a $\varphi \in \Phi_{P}$ there exists a sequence $\left(x_{n}\right)_{n \in \mathbb{N}}$ in $X$ such that

$$
\sup _{k \in \mathbb{N}} p\left(x_{n+1+k}, x_{n+1}\right) \leq a_{n+1} \leq \varphi\left(a_{n}\right), \quad n \in \mathbb{N}, \text { for a sequence }\left(a_{n}\right)_{n \in \mathbb{N}}
$$

and $\lim _{n \rightarrow \infty} p\left(F\left(x_{n}\right), x_{n+1}\right)=0$. Then $x=\lim _{n \rightarrow \infty} x_{n}$ in $(X, d)$ is a fixed point of $F$, and $x \in$ $\operatorname{Ker} p$.

Proof Clearly, $\left(a_{n}\right)_{n \in \mathbb{N}}$ converges to zero as $\varphi \in \Phi_{P}$. Therefore, $\left(x_{n}\right)_{n \in \mathbb{N}}$ is a Cauchy sequence (see (2)) and it converges in $(X, d)$, say, to $x \in \operatorname{Ker} p(X$ is 0 -complete). There exist $y_{n} \in F\left(x_{n}\right)$ such that $\lim _{n \rightarrow \infty} p\left(y_{n}, x_{n+1}\right)=0$. Now, from

$$
p\left(y_{n}, x_{n}\right) \leq p\left(y_{n}, x_{n+1}\right)+p\left(x_{n+1}, x_{n}\right)
$$

and condition (8) it follows that $x \in F(x)$.

Theorem 21, with $\varphi(\alpha)=k \alpha, \alpha \geq 0$ (for $k<1$ ), is an extension of the Nadler theorem on multivalued contractions [14, Theorem 5].

Now, Theorem 21 and Theorem 11 yield the following.

Theorem 22 Let $(X, p)$ be a 0-complete partial metric space, and let $\mathcal{F}$ be a family of mappings $X \rightarrow 2^{X}$ with 0 -closed graphs. Assume that $\varphi \in \Phi_{P}$; some and at least all different $G, H \in \mathcal{F}$ satisfy (12). If for an $F \in \mathcal{F}$ there exists a sequence $\left(x_{n}\right)_{n \in \mathbb{N}}$ such that $\lim _{n \rightarrow \infty} p\left(F\left(x_{n}\right), x_{n+1}\right)=0$ and (17) holds, then all members of $\mathcal{F}$ have the same nonempty set of fixed points; this set is closed in $(X, d)$ and contained in $\operatorname{Ker} p$. 
A simple consequence of Theorem 21 is the following one.

Theorem 23 Let $(X, p)$ be a 0 -complete partial metric space, and let $f: X \rightarrow X$ be a mapping with a 0 -closed graph (e.g., $f:(X, d) \rightarrow(X, d)$ is continuous). Assume that for a $\varphi \in \Phi_{P}$ and $x_{0}$ the following condition is satisfied:

$$
\begin{aligned}
& \sup _{k \in \mathbb{N}} p\left(f^{n+1+k}\left(x_{0}\right), f^{n+1}\left(x_{0}\right)\right) \\
& \quad \leq a_{n+1} \leq \varphi\left(a_{n}\right), \quad n \in \mathbb{N}, \quad \text { for a sequence }\left(a_{n}\right)_{n \in \mathbb{N}} .
\end{aligned}
$$

Then $x=\lim _{n \rightarrow \infty} f^{n}\left(x_{0}\right)$ in $(X, d)$ is a fixed point of $f$, and $x \in \operatorname{Ker} p$.

Proof For $x_{n}=f^{n}\left(x_{0}\right)\left(\right.$ and $\left.x_{n+1}=f^{n+1}\left(x_{0}\right)\right)$, we have (see (2))

$$
0 \leq \lim _{n \rightarrow \infty} p\left(f\left(x_{n}\right), x_{n+1}\right) \leq \lim _{n \rightarrow \infty} p\left(x_{n+1}, x_{n}\right)=0,
$$

and condition (17) holds.

The next proposition shows that Theorem 23 is related to the well-known theorem of Matkowski [2, Theorem 1.2, p.8].

Proposition 24 Let $(X, p)$ be a partial metric space, and let $f: X \rightarrow X$ be a bounded mapping satisfying

$$
p(f(y), f(x)) \leq \varphi(p(y, x)), \quad x, y \in X
$$

for a nondecreasing mapping $\varphi:[0, \infty) \rightarrow[0, \infty)$. Then condition (18) holds.

Proof Let us adopt $x_{n}=f^{n}\left(x_{0}\right)$ and $a_{n}=\sup _{k \in \mathbb{N}} p\left(x_{n+k}, x_{n}\right), n \in \mathbb{N}$. For each $k, n \in \mathbb{N}$, we have

$$
\begin{aligned}
p\left(x_{n+1+k}, x_{n+1}\right) & =p\left(f\left(x_{n+k}\right), f\left(x_{n}\right)\right) \leq \varphi\left(p\left(x_{n+k}, x_{n}\right)\right) \\
& \leq \varphi\left(\sup _{k \in \mathbb{N}} p\left(x_{n+k}, x_{n}\right)\right)=\varphi\left(a_{n}\right)
\end{aligned}
$$

as $\varphi$ is nondecreasing, and we get (18).

Let $(X, p)$ be a partial metric space, and let $f: X \rightarrow X$ be a mapping. Let us recall the conditions used by Romaguera in [15]:

$$
p(f(y), f(x)) \leq \varphi\left(m_{f}(y, x)\right), \quad x, y \in X
$$

for

$$
m_{f}(y, x)=\max \{p(y, x), p(f(y), y), p(f(x), x)\},
$$

and

$$
p(f(y), f(x)) \leq \varphi\left(p_{f}(y, x)\right), \quad x, y \in X
$$


for

$$
p_{f}(y, x)=\max \{p(y, x), p(f(y), y), p(f(x), x),[p(f(y), x)+p(f(x), y)] / 2\} .
$$

For nondecreasing $\varphi$, Ćirić's condition (21) is more general than (20), and (20) is more general than condition (19). All these conditions are used to prove that $f$ has a fixed point. Let us note that $\varphi(0)$ for conditions (19), (20), (21) can be arbitrary as $p(f(x), x)=0$ on the right-hand side of any of these inequalities (for $y=f(x)$ ) means that $x$ is a fixed point of $f$.

Many fixed point theorems use more sophisticated conditions than (19), (20), (21) (see, e.g., [16], [17]) or the spaces under consideration have a richer structure (see, e.g., [9], [18]). We are interested in extending the most classical results.

The subsequent two lemmas are proved for condition (21), and the reasonings for (20) and (19) as well can be easily deduced. The next lemma (for condition (21)) has much in common with Romaguera's Lemma 1 and Lemma 2 [15].

Lemma 25 Let $(X, p)$ be a partial metric space, and let $f: X \rightarrow X$ be a mapping satisfying condition (19), (20), or (21) for a $\varphi \in \Phi_{P}$. Then, for any $x \in X$, the condition

$$
p\left(f^{2}(x), f(x)\right) \leq \varphi(p(f(x), x))
$$

is satisfied, and $\lim _{n \rightarrow \infty} p\left(f^{n+1}(x), f^{n}(x)\right)=0$.

Proof For notational simplicity, let us adopt $x_{n}=f^{n}(x), n \in \mathbb{N}$. For $y=x_{1}$, condition (21) has the following form:

$$
p\left(x_{2}, x_{1}\right) \leq \varphi\left(p_{f}\left(x_{1}, x\right)\right)=\varphi\left(\max \left\{p\left(x_{1}, x\right), p\left(x_{2}, x_{1}\right),\left[p\left(x_{2}, x\right)+p\left(x_{1}, x_{1}\right)\right] / 2\right\}\right) .
$$

We have

$$
\begin{aligned}
p\left(x_{2}, x\right)+p\left(x_{1}, x_{1}\right) & \leq p\left(x_{2}, x_{1}\right)+p\left(x_{1}, x\right)-p\left(x_{1}, x_{1}\right)+p\left(x_{1}, x_{1}\right) \\
& =p\left(x_{2}, x_{1}\right)+p\left(x_{1}, x\right)
\end{aligned}
$$

and therefore,

$$
\max \left\{p\left(x_{2}, x_{1}\right), p\left(x_{1}, x\right)\right\} \leq m_{f}\left(x_{1}, x\right) \leq p_{f}\left(x_{1}, x\right) \leq \max \left\{p\left(x_{2}, x_{1}\right), p\left(x_{1}, x\right)\right\}
$$

holds, i.e., $p_{f}(f(x), x)=\max \left\{p\left(f^{2}(x), f(x)\right), p(f(x), x)\right\}$ (Lemma 1 [15]). This last equality and condition (21) for $p\left(x_{1}, x\right)<p\left(x_{2}, x_{1}\right)$ yield

$$
0<p\left(x_{2}, x_{1}\right) \leq \varphi\left(p_{f}\left(x_{1}, x\right)\right)=\varphi\left(p\left(x_{2}, x_{1}\right)\right)
$$

i.e., $p\left(x_{2}, x_{1}\right)=p\left(f^{2}(x), f(x)\right)=0(\varphi \in \Phi)$. This contradiction proves that $p\left(x_{2}, x_{1}\right) \leq p\left(x_{1}, x\right)$ must hold, and then condition (21) yields $p\left(f^{2}(x), f(x)\right) \leq \varphi(p(f(x), x))$ (see Romaguera's Lemma 2 [15]). Now, it is clear that for arbitrary $x_{0} \in X$ and $x_{n}=f^{n}\left(x_{0}\right)$ the sequence 
$\left(a_{n}\right)_{n \in \mathbb{N}}$, where $a_{n}=p\left(x_{n+1}, x_{n}\right), n \in \mathbb{N}$, converges to zero as $\varphi \in \Phi_{P}$ and $a_{n+1} \leq \varphi\left(a_{n}\right)$ holds.

The next lemma is also helpful in proving fixed point theorems.

Lemma 26 Let $(X, p)$ be a 0-complete partial metric space, and let $f: X \rightarrow X$ be a mapping satisfying condition (19), (20), or (21) for a $\varphi \in \Phi$. If for $x_{n}=f^{n}\left(x_{0}\right), \lim _{m, n \rightarrow \infty} p\left(x_{n}, x_{m}\right)=0$ holds, then $\left(x_{n}\right)_{n \in \mathbb{N}}$ converges in $(X, d)$ to a unique fixed point off, and this point belongs to $\operatorname{Ker} p$.

Proof From $\lim _{m, n \rightarrow \infty} p\left(x_{n}, x_{m}\right)=0$ it follows that $\left(x_{n}\right)_{n \in \mathbb{N}}$ converges to a point $x \in \operatorname{Ker} p$ in $(X, d)$ (Corollary 4). We get

$$
\begin{aligned}
p(f(x), x) \leq & p\left(x_{n+1}, f(x)\right)+p\left(x_{n+1}, x\right) \leq \varphi\left(p_{f}\left(x_{n}, x\right)\right)+p\left(x_{n+1}, x\right) \\
= & \varphi\left(\max \left\{p\left(x_{n}, x\right), p\left(x_{n+1}, x_{n}\right), p(f(x), x),\left[p\left(x_{n+1}, x\right)+p\left(f(x), x_{n}\right)\right] / 2\right\}\right) \\
& +p\left(x_{n+1}, x\right) .
\end{aligned}
$$

Suppose $0<p(f(x), x)$. Then from

$$
p\left(f(x), x_{n}\right) / 2 \leq\left[p(f(x), x)+p\left(x, x_{n}\right)\right] / 2,
$$

and $\lim _{n \rightarrow \infty} p\left(x, x_{n}\right)=\lim _{n \rightarrow \infty} p\left(x, x_{n+1}\right)=0$ it follows that

$$
\max \left\{p\left(x_{n}, x\right), p\left(x_{n+1}, x_{n}\right), p(f(x), x),\left[p\left(x_{n+1}, x\right)+p\left(f(x), x_{n}\right)\right] / 2\right\}=p(f(x), x)
$$

for large $n$. Now we get

$$
0<p(f(x), x) \leq \varphi(p(f(x), x))
$$

a contradiction. Clearly, $p(f(x), x)=0$ means that $p(x, x)=p(f(x), f(x))=p(f(x), x)=0$ (see (2)), and $f(x)=x$ (see (7)). If $x, y$ are fixed points of $f$, then $p(y, y) \leq p(y, x) \leq \varphi(p(y, x))$ (see (2), (21)) means that $x=y$.

The next result extends Romaguera's Theorem 4 [15], and consequently, an earlier celebrated result due to Matkowski [2, Theorem 1.2, p.8].

Theorem 27 Let $(X, p)$ be a 0 -complete partial metric space, and let $f: X \rightarrow X$ be a mapping satisfying condition (19) or (20) for a $\varphi \in \Phi_{P}$ such that

$$
\limsup _{\beta \rightarrow \alpha^{-}} \varphi(\beta)<\alpha, \quad \alpha>0
$$

holds (e.g., if $\varphi$ is nondecreasing). Then $f$ has a unique fixed point; if $x=f(x)$, then $x \in \operatorname{Ker} p$ and $x=\lim _{n \rightarrow \infty} f^{n}\left(x_{0}\right)$ in $(X, d), x_{0} \in X$.

Proof In view of Lemmas 25,26 it is sufficient to prove that $\left(x_{n}\right)_{n \in \mathbb{N}}$ is a Cauchy sequence for $x_{n}=f^{n}\left(x_{0}\right), n \in \mathbb{N}$. Suppose that there are infinitely many $k, n \in \mathbb{N}$ such that 
$p\left(f^{n+1+k}\left(x_{0}\right), f^{k}\left(x_{0}\right)\right) \geq \epsilon>0$. Let $n=n(k)>0$ be the smallest numbers satisfying this inequality. For simplicity let us adopt $x=f^{k}\left(x_{0}\right)$ and $x_{n}=f^{n}(x), n \in \mathbb{N}$. We have

$$
\epsilon \leq p\left(x_{n+1}, x\right) \leq p\left(x_{n+1}, x_{n}\right)+p\left(x_{n}, x\right)<p\left(x_{n+1}, x_{n}\right)+\epsilon,
$$

which for $n=n(k)$ means that

$$
\lim _{k \rightarrow \infty} p\left(x_{n+1}, x\right)=\lim _{k \rightarrow \infty} p\left(x_{n}, x\right)=\epsilon
$$

as we have $\lim _{k \rightarrow \infty} p\left(x_{n+1}, x_{n}\right)=\lim _{k \rightarrow \infty} p\left(x_{1}, x\right)=0$. Now, for $y=x_{n}$, condition (20) yields

$$
\begin{aligned}
\epsilon & \leq p\left(x_{n+1}, x\right) \leq p\left(x_{n+1}, x_{1}\right)+p\left(x_{1}, x\right) \leq \varphi\left(m_{f}\left(x_{n}, x\right)\right)+p\left(x_{1}, x\right) \\
& =\varphi\left(\max \left\{p\left(x_{n}, x\right), p\left(x_{n+1}, x_{n}\right), p\left(x_{1}, x\right)\right\}\right)+p\left(x_{1}, x\right),
\end{aligned}
$$

and we obtain (from (19) as well)

$$
\epsilon \leq \varphi\left(p\left(x_{n}, x\right)\right)+p\left(x_{1}, x\right)
$$

for large $k$. Now, $p\left(x_{n}, x\right)<\epsilon, \lim _{k \rightarrow \infty} p\left(x_{n}, x\right)=\epsilon$, and condition (22) yield

$$
\epsilon \leq \limsup _{k \rightarrow \infty} \varphi\left(p\left(x_{n}, x\right)\right)<\epsilon
$$

a contradiction. Therefore, $\left(x_{n}\right)_{n \in \mathbb{N}}$ is a Cauchy sequence.

Let $\varphi_{1}, \varphi_{2} \in \Phi_{0}$ be continuous mappings. Then $\varphi \in \Phi_{0}$ such that $\varphi=\varphi_{1}$ on $Q \cap[0, \infty)$ and $\varphi=\varphi_{2}$ on $[0, \infty) \backslash Q$ is a member of $\Phi_{P}$ and $\varphi$ satisfies conditions (15), (22); clearly, $\varphi$ does not necessarily belong to $\Phi_{M}$ or to $\Phi_{B W}$.

Paesano and Vetro [9] have proved some theorems on coincidences and common fixed points. Maybe Theorem 27 can be extended to that case.

The next result extends Romaguera's Theorem 3 [15], and consequently, an earlier celebrated result due to Boyd-Wong [1, Theorem 1]. Let us recall that $\varphi(0)=0$ does not spoil the generality of condition (21). The proof is a modification of the one presented by Romaguera.

Theorem 28 Let $(X, p)$ be a 0 -complete partial metric space, and let $f: X \rightarrow X$ be a mapping satisfying condition (21) for a mapping $\varphi \in \Phi_{0}$ and satisfying (15) (e.g., with $\varphi$ upper semicontinuous from the right). Then $f$ has a unique fixed point; if $x=f(x)$, then $x \in \operatorname{Ker} p$ and $x=\lim _{n \rightarrow \infty} f^{n}\left(x_{0}\right)$ in $(X, d), x_{0} \in X$.

Proof We follow the initial part of the proof of Theorem 27 preceding the sentence with condition (20) (Proposition 16 yields $\varphi \in \Phi_{P}$ ). For large $k \in \mathbb{N}$ and $n=n(k)$, we obtain

$$
\begin{aligned}
\epsilon & \leq p\left(x_{n+1}, x\right) \leq p_{f}\left(x_{n+1}, x\right)=\max \left\{p\left(x_{n+1}, x\right),\left[p\left(x_{n+2}, x\right)+p\left(x_{n}, x_{1}\right)\right] / 2\right\} \\
& \leq \max \left\{p\left(x_{n+1}, x\right),\left[p\left(x_{n+2}, x_{n+1}\right)+p\left(x_{n+1}, x\right)+p\left(x_{n}, x\right)+p\left(x_{1}, x\right)\right] / 2\right\},
\end{aligned}
$$


and $\lim _{k \rightarrow \infty} p_{f}\left(x_{n+1}, x\right)=\epsilon$. Now, condition (21) yields

$$
\begin{aligned}
\epsilon & \leq p\left(x_{n+1}, x\right) \leq p\left(x_{n+1}, x_{n+2}\right)+p\left(x_{n+2}, x_{1}\right)+p\left(x_{1}, x\right) \\
& \leq p\left(x_{n+1}, x_{n+2}\right)+\varphi\left(p_{f}\left(x_{n+1}, x\right)\right)+p\left(x_{1}, x\right)
\end{aligned}
$$

and we obtain (see (15))

$$
\epsilon \leq \limsup _{k \rightarrow \infty} \varphi\left(p_{f}\left(x_{n+1}, x\right)\right)<\epsilon,
$$

a contradiction. Therefore, $\left(x_{n}\right)_{n \in \mathbb{N}}$ is a Cauchy sequence.

The next lemma enables us to extend the preceding two theorems.

Lemma 29 Let $f: X \rightarrow X$ be a mapping such that $f^{t}$ for $t \in \mathbb{N}$ has a unique fixed point, say, $x$. Then $x$ is the unique fixed point of $f$. If, in addition, $x \in \lim _{n \rightarrow \infty}\left(f^{t}\right)^{n}\left(x_{0}\right), x_{0} \in X$, then $x \in \lim _{n \rightarrow \infty} f^{n}\left(x_{0}\right), x_{0} \in X$ holds.

Proof If $x$ is a fixed point of $f^{t}$, then $f^{t}(f(x))=f\left(f^{t}(x)\right)=f(x)$ means that $f(x)$ is a fixed point of $f^{t}$ and the uniqueness yields $f(x)=x$. If $x, y \in X$ are fixed points of $f$, then we get $x=f^{t}(x), y=f^{t}(y)$, and $x=y$ as $f^{t}$ has a unique fixed point. If $x \in \lim _{n \rightarrow \infty}\left(f^{t}\right)^{n}\left(x_{0}\right)$ holds for each $x_{0} \in X$, then we also obtain $x \in \lim _{n \rightarrow \infty}\left(f^{t}\right)^{n}\left(f\left(x_{0}\right)\right) \cap \cdots \cap \lim _{n \rightarrow \infty}\left(f^{t}\right)^{n}\left(f^{t-1}\left(x_{0}\right)\right)$, which means that $x \in \lim _{n \rightarrow \infty} f^{n}\left(x_{0}\right)$.

Theorem 30 Let $(X, p)$ be a 0-complete partial metric space, and let $f: X \rightarrow X$ be a mapping satisfying condition (19) or (20) with $f$ replaced by $f^{t}$ for a $t \in \mathbb{N}$, and a $\varphi \in \Phi_{P}$ having property (22) (e.g., with $\varphi$ nondecreasing). Then $f$ has a unique fixed point; if $x=f(x)$, then $x \in \operatorname{Ker} p$ and $x=\lim _{n \rightarrow \infty} f^{n}\left(x_{0}\right)$ in $(X, d), x_{0} \in X$.

Proof Clearly, all the assumptions of Theorem 27 are satisfied for $f$ replaced by $f^{t}$. Now, we apply Lemma 29.

The next theorem is a consequence of Theorem 28 and of Lemma 29.

Theorem 31 Let $(X, p)$ be a 0-complete partial metric space, and let $f: X \rightarrow X$ be a mapping satisfying the condition

$$
p\left(f^{t}(y), f^{t}(x)\right) \leq \max \left\{p(y, x), p\left(f^{t}(y), y\right), p\left(f^{t}(x), x\right),\left[p\left(f^{t}(y), x\right)+p\left(f^{t}(x), y\right)\right] / 2\right\}
$$

for $t \in \mathbb{N}$, and a mapping $\varphi \in \Phi_{0}$ satisfying (15) (e.g., with $\varphi$ upper semicontinuous from the right). Then $f$ has a unique fixed point; if $x=f(x)$, then $x \in \operatorname{Ker} p$ and $x=\lim _{n \rightarrow \infty} f^{n}\left(x_{0}\right)$ in $(X, d), x_{0} \in X$.

Now, we present the respective versions of conditions (19), (20), (21) for a multivalued mapping $F: X \rightarrow 2^{X}$, where $P$ is defined by (11).

$$
\begin{aligned}
& P(F(y), F(x)) \leq \varphi(p(y, x)), \quad x, y \in X, \\
& P(F(y), F(x)) \leq \varphi\left(m_{F}(y, x)\right), \quad x, y \in X
\end{aligned}
$$


for

$$
m_{F}(y, x)=\max \{p(y, x), p(F(y), y), p(F(x), x)\}
$$

and

$$
P(F(y), F(x)) \leq \varphi\left(p_{F}(y, x)\right), \quad x, y \in X
$$

for

$$
p_{F}(y, x)=\max \{p(y, x), p(F(y), y), p(F(x), x),[p(F(y), x)+p(F(x), y)] / 2\} .
$$

For nondecreasing $\varphi$, condition (26) is more general than (25), and (25) is more general than condition (24).

The subsequent two lemmas are proved for condition (26), and the reasonings for (25) and (24) as well can be easily deduced.

At first, let us present the following extension of Lemma 25.

Lemma 32 Let $(X, p)$ be a partial metric space, and let $F: X \rightarrow 2^{X}$ be a mapping. Then

$$
m_{F}(y, x)=p_{F}(y, x)=\max \{p(y, x), p(F(y), y)\}, \quad x \in X, y \in F(x)
$$

holds, and condition (26) for any $\varphi \in \Phi$ yields $p_{F}(y, x)=p(y, x), x \in X, y \in F(x)$ ( $m_{F}$ in place of $p_{F}$ for (25)), and

$$
p(F(y), y) \leq \varphi(p(y, x)), \quad x \in X, y \in F(x) .
$$

If $\varphi \in \Phi_{P}$ and

$$
\begin{array}{r}
\text { for each } y \in F(x), \text { there exists } z \in F(y) \\
\text { such that } p(z, y) \leq \varphi(p(y, x)), x \in X
\end{array}
$$

holds (e.g., $F$ satisfies (28) and is compact valued), then there exists $x_{n+1} \in F\left(x_{n}\right), n \in \mathbb{N}$, such that

$$
p\left(x_{n+2}, x_{n+1}\right) \leq \varphi\left(p\left(x_{n+1}, x_{n}\right)\right), \quad n \in \mathbb{N}, \quad \text { and } \lim _{n \rightarrow \infty} p\left(x_{n+1}, x_{n}\right)=0 .
$$

Proof For $y \in F(x)$, we have (see (2))

$$
\begin{aligned}
p(F(y), x)+p(F(x), y) & =\inf \{p(z, x): z \in F(y)\}+\inf \{p(z, y): z \in F(x)\} \\
& \leq \inf \{p(z, y)+p(y, x)-p(y, y): z \in F(y)\}+p(y, y) \\
& =p(F(y), y)+p(y, x) .
\end{aligned}
$$

Consequently, we get

$$
\begin{aligned}
m_{F}(y, x) & \leq p_{F}(y, x) \leq \max \{p(y, x), p(F(y), y), p(F(x), x),[p(F(y), y)+p(y, x)] / 2\} \\
& \leq \max \{p(y, x), p(F(y), y)\}
\end{aligned}
$$


as $p(F(x), x) \leq p(y, x), y \in F(x)$ holds. Clearly,

$$
\max \{p(y, x), p(F(y), y)\} \leq m_{F}(y, x) \leq p_{F}(y, x)
$$

is satisfied and hence we get (27). Suppose $p(y, x)<p(F(y), y)$ for a $y \in F(x)$. Then (see (26))

$$
p(F(y), y) \leq P(F(y), F(x)) \leq \varphi\left(p_{F}(y, x)\right)=\varphi(p(F(y), y))
$$

means that $p(F(y), y)=0($ as $\varphi \in \Phi)$, and $p(y, x)=p(F(y), y)$. This contradiction proves (28).

Let $x_{1} \in X, x_{2} \in F\left(x_{1}\right)$ be arbitrary. If $x_{n+1}, x_{n}$ are known, then let $x_{n+2} \in F\left(x_{n+1}\right)$ be such that (see (29) or (28) and Proposition 12) $p\left(x_{n+2}, x_{n+1}\right) \leq \varphi\left(p\left(x_{n+1}, x_{n}\right)\right)$. If $p \in \Phi_{P}$, then for $a_{n}=p\left(x_{n+1}, x_{n}\right)$ we get $\lim _{n \rightarrow \infty} a_{n}=0$.

Now, let us prove an analog of Lemma 26.

Lemma 33 Let $(X, p)$ be a 0 -complete partial metric space, and let $F: X \rightarrow 2^{X}$ be a mapping with 0-closed graph satisfying condition (24), (25), or (26) for a $\varphi \in \Phi$. If $\lim _{n \rightarrow \infty} p\left(F\left(x_{n}\right), x_{n+1}\right)=0$ and $\lim _{m, n \rightarrow \infty} p\left(x_{n}, x_{m}\right)=0$, then $\left(x_{n}\right)_{n \in \mathbb{N}}$ converges in $(X, d)$ to a fixed point of $F$, and this point belongs to $\operatorname{Ker} p$.

Proof From $\lim _{m, n \rightarrow \infty} p\left(x_{n}, x_{m}\right)=0$ it follows that $\left(x_{n}\right)_{n \in \mathbb{N}}$ converges to a point $x \in \operatorname{Ker} p$ in $(X, d)$ (Corollary 4). We get

$$
\begin{aligned}
p(F(x), x) \leq & p\left(F(x), x_{n+1}\right)+p\left(x_{n+1}, x\right) \\
\leq & P\left(F(x), F\left(x_{n}\right)\right)+p\left(x_{n+1}, x\right) \leq \varphi\left(p_{F}\left(x_{n}, x\right)\right)+p\left(x_{n+1}, x\right) \\
= & \varphi\left(\max \left\{p\left(x_{n}, x\right), p\left(x_{n+1}, x_{n}\right), p(F(x), x),\left[p\left(x_{n+1}, x\right)+p\left(F(x), x_{n}\right)\right] / 2\right\}\right) \\
& +p\left(x_{n+1}, x\right) .
\end{aligned}
$$

Suppose $0<p(F(x), x)$. Then from

$$
p\left(F(x), x_{n}\right) / 2 \leq\left[p(F(x), x)+p\left(x, x_{n}\right)\right] / 2,
$$

and $\lim _{n \rightarrow \infty} p\left(x, x_{n}\right)=\lim _{n \rightarrow \infty} p\left(x, x_{n+1}\right)=0$ it follows that

$$
\max \left\{p\left(x_{n}, x\right), p\left(x_{n+1}, x_{n}\right), p(F(x), x),\left[p\left(x_{n+1}, x\right)+p\left(F(x), x_{n}\right)\right] / 2\right\}=p(F(x), x)
$$

for large $n$. Now we get

$$
0<p(F(x), x) \leq \varphi(p(F(x), x))
$$

a contradiction. Clearly, $p(F(x), x)=0$ means that $x \in \operatorname{Ker} p$ (see (2)). There exist $y_{n} \in F\left(x_{n}\right)$ such that $\lim _{n \rightarrow \infty} p\left(y_{n}, x_{n+1}\right)=0$, and now,

$$
p\left(y_{n}, x_{n}\right) \leq p\left(y_{n}, x_{n+1}\right)+p\left(x_{n+1}, x_{n}\right)
$$

together with condition (8) yield $x \in F(x)$. 
It should be noted that a partial metric $p$ defines metric $\delta$ in the following way: $\delta(x, y)=0$ iff $x=y$, and $\delta(x, y)=p(x, y)$ for $x \neq y$. The topology of $(X, \delta)$ is clearly larger than the topology of $(X, d)$ (see (7)). Moreover, $(X, p)$ is 0-complete iff $(X, \delta)$ is complete [19], [20, Proposition 2.1].

If the proof of a theorem is based on $p\left(x_{n+1}, x_{n}\right) \neq 0, n \in \mathbb{N}$, then it works for $(X, \delta)$ and the theorem is an immediate consequence of the respective result (if known) for metric spaces. Numerous examples can be found in [20].

Let us add that Corollary 4 and Proposition 5 show that for a 0 -complete partial metric space $(X, p)$, if we prove that $\lim _{m, n \rightarrow \infty} p\left(x_{n}, x_{m}\right)=0$, then the remaining part of the proof concerns the metric space $(X, d)$ (see $(7))$. Let us also recall that Ker $p$ with its partial metric topology is a closed metric subspace of $(X, d)[6$, Lemma 2.2].

\section{Competing interests}

The author declares that they have no competing interests.

\section{Acknowledgements}

The work has been supported by the Polish Ministry of Science and Higher Education.

Received: 23 April 2014 Accepted: 12 August 2014 Published: 02 Sep 2014

\section{References}

1. Boyd, DW, Wong, JSW: On nonlinear contractions. Proc. Am. Math. Soc. 20, 458-464 (1969)

2. Matkowski, J: Integrable solutions of functional equations. Diss. Math. 127, 1-68 (1975)

3. Matthews, SG: Partial metric topology. Proc. 8th Summer Conference on General Topology and Applications. Ann. N.Y. Acad. Sci. 728, 183-197 (1994)

4. Oltra, S, Valero, O: Banach's fixed point theorem for partial metric spaces. Rend. Ist. Mat. Univ. Trieste 36, 17-26 (2004)

5. O'Neill, SJ: Partial metrics, valuations, and domain theory. Proc. 11th Summer Conference on General Topology and Applications. Ann. N.Y. Acad. Sci. 806, 304-315 (1996)

6. Pasicki, L: Partial metric, fixed points, variational principles. Fixed Point Theory. (to appear)

7. Romaguera, S: A Kirk type characterization of completeness for partial metric spaces. Fixed Point Theory Appl. 2010 Article ID 493298 (2010)

8. Paesano, D, Vetro, P: Suzuki's type characterizations of completeness for partial metric spaces and fixed points for partially ordered metric spaces. Topol. Appl. 159, 911-920 (2012)

9. Paesano, D, Vetro, P: Common fixed points in a partially ordered partial metric space. Int. J. Anal. 2013, Article ID $428561(2013)$

10. Aydi, H, Abbas, M, Vetro, C: Partial Hausdorff metric and Nadler's fixed point theorem on partial metric spaces. Topol. Appl. 159, 3234-3242 (2012)

11. Pasicki, L: Towards lim. Topol. Appl. 158, 479-483 (2011)

12. Ćirić, L, Samet, B, Aydi, H, Vetro, C: Common fixed points of generalized contractions on partial metric spaces. Appl. Math. Comput. 218, 2398-2406 (2011)

13. Matkowski, J: Fixed point theorems for mappings with a contractive iterate at a point. Proc. Am. Math. Soc. 62, 344-348 (1977)

14. Nadler, SB: Multi-valued contraction mappings. Pac. J. Math. 30, 475-488 (1969)

15. Romaguera, S: Fixed point theorems for generalized contractions on partial metric spaces. Topol. Appl. 159, 194-199 (2012)

16. Altun, I, Acar, Ö: Fixed point theorems for weak contractions in the sense of Berinde on partial metric spaces. Topol. Appl. 159, 2642-2648 (2012)

17. Alghamdi, MA, Shahzad, N, Valero, O: On fixed point theory in partial metric spaces. Fixed Point Theory Appl. 2012, 175 (2012)

18. Alghamdi, MA, Shahzad, N, Valero, O: Fixed point theorems in general metric spaces with applications to computer science. Fixed Point Theory Appl. 2013, 118 (2013)

19. Hitzler, P, Seda, A: Mathematical Aspects of Logic Programming. Semantics, Studies in Informatic Series. Chapman \& Hall, CRC Press, Boca Raton (2011)

20. Haghi, RH, Rezapour, S, Shahzad, N: Be careful on partial metric fixed point results. Topol. Appl. 160, $450-454$ (2013)

10.1186/1687-1812-2014-185

Cite this article as: Pasicki: Fixed point theorems for contracting mappings in partial metric spaces. Fixed Point Theory and Applications 2014, 2014:185 